\title{
Entrainment in a Dense Current Flowing Down a Rough Sloping Bottom in a Rotating Fluid ${ }^{\mathscr{O}}$
}

\author{
LUISA OTTOLENGHI \\ University of Roma Tre, Rome, Italy \\ Claudia Cenedese \\ Woods Hole Oceanographic Institution, Woods Hole, Massachusetts \\ Claudia Adduce \\ University of Roma Tre, Rome, Italy
}

(Manuscript received 28 July 2016, in final form 8 November 2016)

\begin{abstract}
Dense oceanic overflows descend over the rough topography of the continental slope entraining and mixing with surrounding waters. The associated dilution dictates the fate of these currents and thus is of fundamental importance to the formation of deep water masses. The entrainment in a dense current flowing down a sloping bottom in a rotating homogeneous fluid is investigated using laboratory experiments, focusing on the influence of the bottom roughness on the flow dynamics. The roughness is idealized by an array of vertical rigid cylinders and both their spacing and height are varied as well as the inclination of the sloping bottom. The presence of the roughness is generally observed to decelerate the dense current, with a consequent reduction of the Froude number, when compared to the smooth bottom configuration. However, the dilution of the dense current due to mixing with the ambient fluid is enhanced by the roughness elements, especially for low Froude numbers. When the entrainment due to shear instability at the interface between the dense current and the ambient fluid is low, the additional turbulence and mixing arising at the bottom of the dense current due to the roughness elements strongly affects the dilution of the current. Finally, a strong dependence of the entrainment parameter on the Reynolds number is observed.
\end{abstract}

\section{Introduction}

Dense currents are flows driven by the difference in density between two fluids due to a gradient in the temperature or the concentration fields. In the environment, these kinds of flows widely occur both in the atmosphere (e.g., sea breeze, katabatic currents) and in the oceans (i.e., oceanic overflows) [see Simpson (1997) for a review].

Overflows are dense water masses intruding into a lighter ambient fluid and often moving over a sill or

Supplemental information related to this paper is available at the Journals Online website: http://dx.doi.org/10.1175/ JPO-D-16-0175.s1.

Corresponding author e-mail: Luisa Ottolenghi, luisa.ottolenghi@ uniroma3.it through a bathymetric constriction. Sources of these dense water masses are generally located at high latitudes where the strong atmospheric cooling causes a temperature reduction, the formation of ice, and brine rejection, which induces an increase in salinity. One example is Nordic Seas waters flowing into the North Atlantic Ocean through Denmark Strait (Girton and Sanford 2003) and the Faroe Bank Channel (Mauritzen et al. 2005), forming North Atlantic Deep Water (NADW), a fundamental component of the thermohaline circulation. Generation of dense waters is also observed in marginal seas where evaporation causes an increase in density, for example, the Mediterranean waters flowing into the Atlantic Ocean through the Gibraltar Strait (Price et al. 1993) or the Red and the Persian Sea waters spreading into the Arabian Sea (Peters et al. 2005). Oceanic overflows are observed to descend over the continental slope until they reach the 
ocean bottom or their level of neutral buoyancy. Overflow proprieties, that is, temperature, salinity, and particle and nutrient concentrations, change during this descent because of entrainment processes, which consequently affect both the local ecosystems as well as the global climate equilibrium through the thermohaline circulation.

Earth's rotation plays a major role in the dynamics of these overflows. One of the most prominent effects is the deflection of the dense current trajectory to the right looking downstream (in the North Hemisphere) due to the Coriolis acceleration. The momentum is mainly in geostrophic balance with the effect of bottom drag resulting in a descent of the current across isobaths. The effect of the entrainment is similar to that of the bottom drag in the momentum balance, but it also modifies the continuity equation (Price and Barringer 1994). When a dense overflow or a turbidity current flows into a submarine channel, transport in the Ekman boundary layers due to Earth's rotation induces a complex traverse circulation that strongly affects the dense current dynamics when the Ekman number is of the order of 1 and the Froude number (Fr) is subcritical (Umlauf and Arneborg 2009a,b). In particular, entrainment can be augmented by this secondary transverse circulation (Umlauf et al. 2010). For larger values of the Ekman number a similar secondary traverse flow was observed in the laboratory (Cossu et al. 2010; Cossu and Wells 2010). Furthermore, when a dense current flows down a canyon it can accelerate increasing the Fr and Reynolds (Re) number of the flow leading to overturning and turbulence and hence an increase of the entrainment rate, as observed in the laboratory study of Wåhlin et al. (2008).

Entrainment processes are fundamental players in the dynamics of dense currents because they contribute to the dilution of the current, hence changing the buoyancy forces driving the motion itself. A faithful representation of overflows in climate and general circulation models requires a correct parameterization of the entrainment, which is not resolved by coarse models. For this reason, entrainment and mixing processes in gravity currents have been the subject of a large number of studies that employ both numerical simulations (e.g., Chang et al. 2005; Ezer 2005; Legg et al. 2006; Xu et al. 2006; Özgökmen et al. 2009; Ottolenghi et al. 2016a,b) and laboratory experiments (e.g., Hacker et al. 1996; Cenedese et al. 2004; Cenedese and Adduce 2008). A widely used entrainment parameterization was proposed by Turner (1986), based on the laboratory experiments of Ellison and Turner (1959). The entrainment rate magnitude is modeled to be a function of the Froude number and is nonzero only for supercritical flows $\left(\mathrm{Fr}^{2}>1.25\right)$. Entrainment for subcritical flows was included in subsequent parameterizations (Cenedese et al. 2004; Cenedese and Adduce 2010) and recently a parameterization depending on both the Froude and Reynolds numbers was proposed by Cenedese and Adduce (2010). Similar Froude (or Richardson) numberbased parameterizations have also been suggested for turbidity currents (Parker et al. 1987).

The existing entrainment parameterizations account primarily for the shear-induced entrainment at the interface between the dense flow and the ambient fluid. However, the turbulence generated by bottom roughness, which produces an enhanced drag, can be intense and may need to be considered (Dallimore et al. 2001; Fernandez and Imberger 2006; Wells et al. 2010). Parameterizations accounting for both the Richardson number ( $\mathrm{Ri}$ ) and the stresses generated at the bottom, directly related to the bed roughness, predict values of the entrainment parameter $E$ that are in a better agreement with field measurements than the values obtained from parameterizations based only on $\mathrm{Ri}$ (Fernandez and Imberger 2006). In particular, the study of Fernandez and Imberger (2006) showed that at relatively high Richardson numbers $(\mathrm{Ri}>10)$ the entrainment rate was underestimated by parameterization written as $E=E(\mathrm{Ri})$, while the entrainment was better predicted using an entrainment formulation in the form $E=E\left(C_{D}, \mathrm{Ri}\right)$ (Hebbert et al. 1979; Dallimore et al. 2001). On the other hand, when $\mathrm{Ri}<10$, all the entrainment laws tested by the authors were in agreement with the entrainment measured in the field. During a flow regime governed by a balance between drag and buoyancy forces, the influence of $\mathrm{Ri}$ decreases when the roughness becomes large and the influence of the bottom stress on the entraining interface increases. Bottom roughness and an oscillating sloping bottom have also been used to investigate the effect of bottom boundary mixing on a dense gravity current flowing down a slope. An increase in background turbulence was observed to increase the height of the dense current and decrease of the front speed (Noh and Fernando 1991, 1992).

In the study of Negretti et al. (2008), it was observed that the rough bottom affects the dynamics of the flow via two contrasting mechanisms: the additional turbulence caused by the rough bottom enhances entrainment, but, at the same time, the rough bottom causes a decrease in the current velocity, inhibiting the formation of large-scale turbulent structures due to shear instability, with a consequent reduction in entrainment at the interface. Similar findings were also presented in the studies of Nogueira et al. (2013, 2014), which focused on lock-release gravity currents flowing over a natural 
rough bed. Using numerical simulations, Özgökmen et al. (2004) found that the entrainment in bottom gravity currents flowing over a complex topography was enhanced when compared to that in a current moving over a smooth surface. As a consequence, bottom roughness influences the overflow product distribution in that the highest density classes are removed and the level of neutral buoyancy is reached higher in the water column (Özgökmen and Fischer 2008). The dynamics of gravity currents flowing over a rough bottom have also been studied in the context of flows within aquatic vegetation (Luhar et al. 2008; Nepf 2012). In these studies, the dimensionless parameter characterizing the roughness elements' distribution was correlated with the size of the turbulent structures arising in the flow. Following Luhar et al. (2008) and Nepf (2012), the frontal area per volume $a$ and the frontal area per bed area $a h$ are defined as $a=d / \Delta S^{2}$, where $d, h$, and $\Delta S$ are the diameter, the height, and the spacing of the roughness elements, respectively. In particular, $\Delta S$ is defined as the distance between the centers of two adjacent elements. In the cited investigations, it was observed that for $a h \geq 0.1$ a shear layer was generated in the flow on top of the roughness elements, with the consequent formation of turbulent structures. These vortices can penetrate to different depths in the roughness region for different values of $a h$. The larger the $a h$, the deeper the penetration of the shear-induced vortices; hence, the homogenization and mixing of the fluid within the roughness elements is regulated by $a h$.

The aim of the present laboratory study is to investigate the effects of a rough bottom on the entrainment in dense currents propagating under conditions similar to those of Cenedese and Adduce (2008). In particular, the entrainment in a dense current flowing down a rough sloping bottom in a rotating environment is examined by varying the inclination of the slope, the height of the roughness elements, and their distribution on the slope. In the ocean, bottom roughness presents a wide variety of scales, from a few tenths of centimeters (e.g., Fernandez and Imberger 2006), to a few tenths of meters (e.g., Hernández-Molina et al. 2006; Hanquiez et al. 2007), and up to a few hundreds of meters when looking at the multiple fractures characterizing the Reykjanes Ridge (e.g., Keeton et al. 1997). The height of the dense gravity currents flowing over these roughness elements is highly variable too, and although the major overflows are several hundreds of meters thick, thin, dense currents can result from the branching of these larger overflows into canyons or around ridges (Sherwin and Turrell 2005) and can be found in marginal basins like the Baltics (Umlauf and Arneborg 2009a,b) but also in lakes (Dallimore et al. 2001; Fernandez and Imberger
2006) and in reservoirs (Hebbert et al. 1979; Fernandez and Imberger 2006). Hence, to cover the wide range of scales characterizing the roughness elements and the thickness of the gravity currents in the ocean, in the current study we investigate gravity currents having a height that will systematically vary from being smaller than the roughness elements, characteristic of flow within aquatic vegetation (e.g., Nepf 2012), to much larger than the roughness elements, as is the case for the larger overflows or overflows in reservoirs, where both the roughness and current height can be small but give a large ratio of current to roughness height (e.g., Fernandez and Imberger 2006).

This paper is organized as follows: In section 2, the experimental apparatus is described together with the experimental technique followed to perform the laboratory experiments. The entrainment parameter and the dimensionless numbers characterizing the flow are defined in sections 3 and 4 , respectively. Sections 5 and 6 discuss the behavior of the dense current as it moves downslope and the different flow regimes observed, respectively. Sections 7 and 8 illustrate the entrainment parameter dependence on the relevant dimensionless numbers. The results are discussed in section 9, and the conclusions are given in section 10 .

\section{Laboratory experiments}

\section{a. Experimental apparatus}

A total of 68 experiments were performed using an experimental technique similar to Cenedese and Adduce (2008). Dense gravity currents were generated in a glass square tank, $60 \mathrm{~cm}$ high and $60 \mathrm{~cm}$ wide, by the constant supply of salted and dyed water at a measured density $\rho_{1}$ into an ambient fluid at density $\rho_{0}<\rho_{1}$ (Fig. 1). The tank was placed on a 1-m-diameter rotating turntable with a constant rotation rate, characterized by a Coriolis parameter $f=1 \mathrm{~s}^{-1}$. A sloping bottom was placed inside the tank forming an angle $\theta$ with the horizontal. The experiments were performed by varying the slope $s=\tan \theta$ between 0.4 and 8 , while the flow rate of dense fluid $Q_{0}=2.5 \mathrm{~cm}^{3} \mathrm{~s}^{-1}$ was kept constant. The dense fluid was pumped through a plastic tube into a small box with a sponge inside, that is, the source of dense water, located on the top-right hand side of the sloping bottom (Fig. 1), at a depth $H_{0}$ from the free surface. The depth $H_{0}$ took values of $10 \mathrm{~cm}$ for $0.4 \leq s \leq 2$ and $5 \mathrm{~cm}$ for $2<s \leq 8$. Small, rigid, vertical cylinders were attached to the slope in order to investigate the effect of a rough bottom on the flow dynamics. The cylinders were characterized by a diameter $d=0.5 \mathrm{~cm}$, 


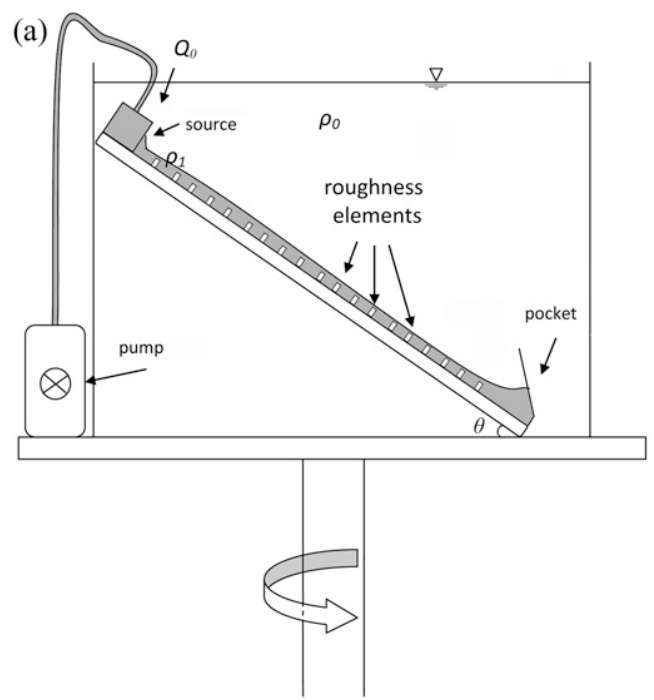

(b)

FIG. 1. (a) Schematic elevation view of the experimental apparatus, not to scale. (b) Camera plan view of the experimental apparatus. Insert is a zoomed in view of the white vertical cylinders.

which was kept fixed; a height $h$, which assumed three values of $0.1,0.3$, and $0.5 \mathrm{~cm}$; and a spacing $\Delta S$, which assumed two values $\Delta S=0.8 \mathrm{~cm}$ (dense configuration) and $\Delta S=1.6 \mathrm{~cm}$ (sparse configuration). In some experiments the cylinders were higher than the current depth, while in others they were smaller (section 7). The smooth configuration, that is, a sloping bottom with no roughness elements, was also tested for all the considered values of $s$ for comparison with Cenedese and Adduce (2008).

The top view of the experiments was recorded in the rotating system of reference by a camera fixed on the top of the rotating table. The duration of each experiment and the time the current took to reach the bottom of the slope were recorded by two stopwatches. After the dense current propagated through and/or over the rough bottom, the dense fluid was collected into a pocket located at the end of the slope and solid vertical walls surrounding the slope assured the absence of lateral leakages (Fig. 1). A more detailed description of the experimental apparatus can be found in Cenedese and Adduce (2008).

\section{b. Description of the experimental procedure}

After the ambient fluid reached solid body rotation, the pump was turned on and the experiment started. The dense current was generated and propagated down the slope through and/or over the rough bottom, entraining ambient fluid and diluting. The trajectory of the dense current was deflected toward the right, looking downslope, by the Coriolis acceleration (see the movie in the supplemental information). The time taken by the dense current to reach the end of the slope $\delta t$ was recorded by one of the two stopwatches. As in Cenedese and Adduce (2008), in order to investigate the entrainment occurring along the descent of the current and minimize the influence of the entrainment occurring near the head of the current, the experiments lasted for a time $\Delta T \gg \delta t$. Hence, the pump continued to provide dense fluid to the system until the dense fluid in the pocket reached a fixed vertical level and then the pump was turned off. The second stopwatch recorded the duration of the entire experiment $\Delta T$.

\section{Entrainment parameter}

The focus of the experiments was to quantify the volume of ambient fluid entrained by the dense current. The volume $V$ of dense fluid collected into the pocket was approximately the same in each experiment, since the dense fluid level reached a fixed height $H$ inside the pocket after the time $\Delta T$. Error bars are evaluated considering the possible variations of the fluid volume collected into the pocket due to the difference between the value of the fixed vertical level $H$ and the real steady height reached by the dense fluid after its adjustment along the width of the tank $( \pm 0.5 \mathrm{~cm})$. The possible errors in the evaluation of $V$ affect all the derived quantities discussed below. The volume $V$ is composed of dense fluid of density $\rho_{1}$ and ambient fluid of density $\rho_{0}$ entrained during the descent of the dense current

$$
V=\left(Q_{e}+Q_{0}\right) \Delta T,
$$


where $Q_{e}$ is the entrainment volume flux into the dense current.

The average entrainment velocity $w_{e}$ is defined as

$$
w_{e}=\frac{Q_{e}}{A}=\frac{1}{A}\left(\frac{V}{\Delta T}-Q_{0}\right),
$$

where $A$ is the area on the slope covered by the dense current, evaluated by an image analysis of the top-view pictures recorded by the camera. The bulk velocity of the dense current $U_{m}$ is defined, following Cenedese and Adduce (2008), as

$$
U_{m}=\frac{L}{\delta t}
$$

where $L$ is the length of the path followed by the current. The entrainment parameter $E$ is finally defined as

$$
E=\frac{w_{e}}{U_{m}}=\frac{\delta t}{L A}\left(\frac{V}{\Delta T}-Q_{0}\right) .
$$

Considering $n=\Delta T / \delta t$ as the hypothetical number of times the dense current has traveled down the slope during each experiment, it is possible to evaluate the mean height of the gravity current $H_{m}$. In particular, the volume of fluid $V$ is considered to be distributed over the area $A$, and taking into account the volume $V_{c}$ occupied by the cylindrical roughness elements (with $V_{c}$ varying for each roughness and depending on $A$ )

$$
H_{m}=\frac{\frac{V \delta t}{\Delta T}+V_{c}}{A}=\frac{\frac{V}{n}+V_{c}}{A} .
$$

During each experiment the height of the dense current, approximately halfway down the slope, was observed and recorded. However, the precision of this measurement was poor and we only used it to have a visual confirmation of the values obtained for $H_{m}$. Furthermore, assuming the fluid in the dense current is homogeneous, the density of the fluid inside the pocket at the end of the experiment is given by

$$
\rho_{p}=\frac{\rho_{1} Q_{0}+\rho_{0} Q_{e}}{Q_{0}+Q_{e}},
$$

and the mean reduced gravity $g_{m}^{\prime}$ of the dense current is defined as

$$
g_{m}^{\prime}=g \frac{\rho_{\text {mean }}-\rho_{0}}{\left(\rho_{1}+\rho_{0}\right) / 2},
$$

where $\rho_{\text {mean }}=\left(\rho_{1}+\rho_{p}\right) / 2$, the mean value between the densities at the beginning and at the end of the slope.

\section{Dimensionless parameters}

The discussion of the dimensionless parameters characterizing the bottom roughness is similar to that of Cenedese et al. (2017), as follows in the next paragraph. The elevation density $\mu$ is defined by

$$
\mu=\frac{A_{E}}{A_{T E}}=\frac{d}{\Delta S}
$$

where $A_{E}$ is the area of the field covered by the cylinders in elevation, as seen by the advancing current, and $A_{T E}$ is the total area of the field in elevation (measured to the top of the cylinders), as shown in Fig. 2a; $\sigma$, the plan density, is defined by

$$
\sigma=\frac{A_{P}}{A_{T P}}=\frac{\pi}{4} \gamma \mu^{2},
$$

where $A_{P}$ is the area of the base covered by the cylinders in plan, $A_{T P}$ is the total area of the base in plan, and $\gamma=\Delta S_{x} / \Delta S_{y}=2 / \sqrt{3}$ for our configuration (Fig. 2b); and $\alpha$, the aspect ratio of the roughness elements, is defined by

$$
\alpha=\frac{h}{d} .
$$

The roughness parameters for the sparse configuration were $\sigma=0.08$ and $\mu=0.30$ and for the dense configuration were $\sigma=0.34$ and $\mu=0.61$. For our particular configuration in which each element is equidistant to the surrounding ones, the parameters $\sigma$ and $\mu$ are not independent; however, this is not the case when the roughness elements are not equidistant from each other. The value of $\alpha$ was $0.2,0.6$, and 1 for each configuration. The above parameters can be combined to give the roughness frontal area per bed area parameter introduced by Nepf (2012):

$$
a h=\gamma \frac{d h}{\Delta S^{2}}=\gamma \alpha \mu^{2}=\frac{4}{\pi} \alpha \sigma .
$$

The values of $a$ and $a h$ for the roughness used are summarized in Table 1. An additional nondimensional number is the ratio of the cylinder height to the current depth

$$
\lambda=\frac{h}{H_{m}},
$$

which in the present experiments varied between 0.08 and 1.47. It is important to note that for $\lambda>1$, the array of cylinders represents a series of obstacles for the current and not a bottom roughness. However, for simplicity, we call the array of cylinders "roughness elements" also for the experiments having $\lambda>1$. 


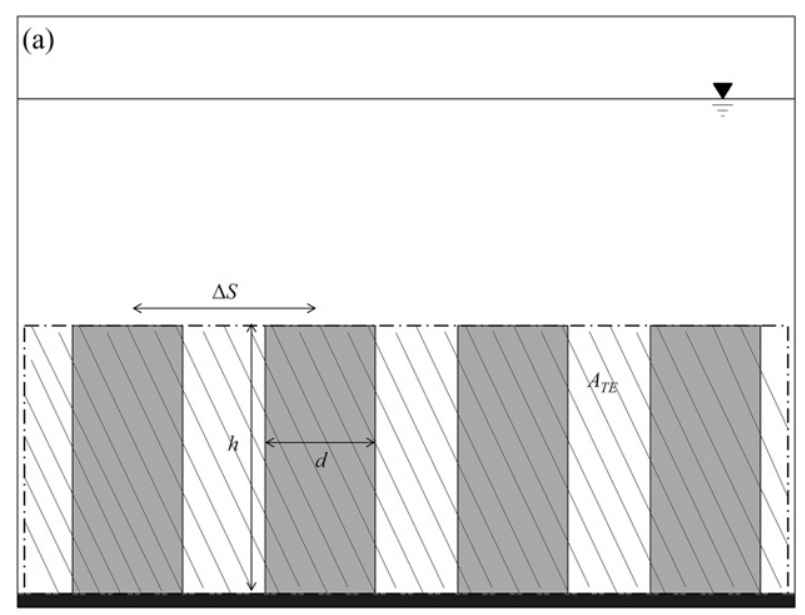

(b)

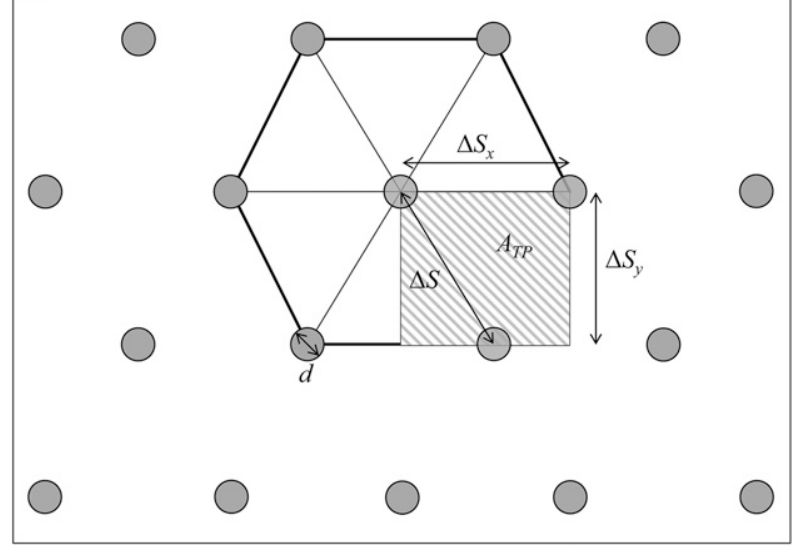

FIG. 2. (a) Schematic elevation view illustrating the area (dashed) of the field measured to the top of the cylinders $A_{T E}$ and the area of the field $A_{E}$ covered by the cylinders (gray); (b) plan view illustrating the area of the base plan $A_{T P}$ (diagonal shading) associated with the area of the base covered by one cylinder $A_{P}$ (gray).

Finally, the Reynolds and the Froude numbers are defined as

$$
\begin{aligned}
& \mathrm{Re}=\frac{H_{m} U_{m}}{\nu}, \text { and } \\
& \mathrm{Fr}=\frac{U_{m}}{\sqrt{g_{m}^{\prime} H_{m} \cos \theta}},
\end{aligned}
$$

with $\nu$ as the kinematic viscosity of water $\left(\nu=10^{-2} \mathrm{~cm}^{2} \mathrm{~s}^{-1}\right)$. The Froude and Reynolds numbers varied between $\mathrm{Fr}=0.43-2.57$ and $\mathrm{Re}=13-134$. It is important to note that the values of the Reynolds number are lower than in similar studies investigating the dynamics of a dense current over a smooth bottom (e.g., Ellison and Turner 1959; Parker et al. 1987; Cenedese and Adduce 2008). Hence, the values of the entrainment parameter $E$ are also expected to be lower than in the studies
TABLE 1. Characterization of the bottom roughness.

\begin{tabular}{cccccc}
\hline \hline Name & Type & $h(\mathrm{~cm})$ & $\Delta S(\mathrm{~cm})$ & $a\left(\mathrm{~cm}^{-1}\right)$ & $a h$ \\
\hline ROUGH1 & Dense & 0.5 & 0.8 & 0.91 & 0.44 \\
ROUGH2 & Sparse & 0.5 & 1.6 & 0.22 & 0.11 \\
ROUGH3 & Dense & 0.3 & 0.8 & 0.91 & 0.27 \\
ROUGH4 & Sparse & 0.3 & 1.6 & 0.22 & 0.07 \\
ROUGH5 & Dense & 0.1 & 0.8 & 0.91 & 0.08 \\
ROUGH6 & Sparse & 0.1 & 1.6 & 0.22 & 0.02 \\
\hline
\end{tabular}

mentioned above, given the Reynolds number dependence of the entrainment parameter (e.g., Cenedese and Adduce 2010).

\section{Downslope flow mechanisms}

In a recent study, Cenedese et al. (2017) investigated a nonrotating, lock-exchange, dense gravity current propagating over a bottom roughness made of an array of vertical rigid cylinders having the same geometrical distribution as in the present study (Fig. 1b). The larger dimension of their apparatus allowed them to investigate the details of the flow between the cylinders, and they observed that when a sparse configuration of roughness elements is present, the dense current propagates between the cylinders that act on the current more as obstacles than as a rough bottom boundary, while for a dense configuration, the dense current rides on top of the cylinders. The experiments of Cenedese et al. (2017) also showed that for a sparse configuration the entrainment is enhanced by the vortices generated in the wake of the cylindrical obstacles, especially when the roughness element height is approximately the same as the dense current interface. Furthermore, in the dense configuration the dense fluid riding on top of the cylinders is located above the lighter ambient water between the cylinders. This configuration is convectively unstable and the dense current fluid is diluted by mixing with the lighter fluid between the cylinders.

The present experiments focused on the effect of bottom roughness on entrainment and the experimental apparatus was too small to clearly detect the details of the fluid propagating between the cylinders. However, for some of the experiments in the sparse configuration, it was clear that the dense fluid propagated between the cylinders, while this behavior was not observed for the experiments having a dense configuration. Hence, we will make the assumption that the different behaviors observed and discussed in detail in Cenedese et al. (2017) are occurring also in our experimental setup: that is, that the dense current propagates between and on top of the cylinders for a sparse and dense configuration, respectively. 
TABLE 2. Frequency of occurrence (\%) of the different flow regimes for selected intervals of Re and $\mathrm{Fr}^{2} \mathrm{Re}$.

\begin{tabular}{lcccccc}
\hline \multicolumn{1}{c}{ Regime } & $\operatorname{Re}<39$ & $39 \leq \operatorname{Re} \leq 85$ & $\operatorname{Re}>85$ & $\operatorname{Fr}^{2} \operatorname{Re}<50$ & $50 \leq \operatorname{Fr}^{2} \operatorname{Re} \leq 180$ & $\operatorname{Fr}^{2} \operatorname{Re}>180$ \\
\hline Laminar and waves & 90 & 15 & 0 & 79.2 & 18.8 & 0 \\
Breaking waves & 10 & 80 & 5.6 & 20.8 & 75 & 6.2 \\
Turbulent & 0 & 5 & 94.4 & 0 & 89.5 \\
\hline
\end{tabular}

The procedure followed to obtain the entrainment parameter $E$, discussed in section 3, utilizes the volume of the dense current at the bottom of the slope and hence does not distinguish between the entrainment of ambient waters through the current interface and the dilution of the current due to convective instability occurring in the dense configuration. Hence, hereafter by "entrainment" we will refer to both of the above processes contributing to the dilution of the dense current.

\section{Flow regimes}

As observed for a rotating dense current over a smooth, sloping bottom (e.g., Cenedese et al. 2004), the dense fluid exiting the source moved first downslope and then it was deflected to the right (looking downslope) by the effect of rotation.

According to Cenedese et al. (2004) and Cenedese and Adduce (2008), different flow regimes can be observed during the descent of a dense current over a sloping bottom. A laminar regime, a wave regime, a breaking-wave regime, or a turbulent regime develop depending on the values of Fr and Re. Cenedese and Adduce (2008) selected specific Re to mark the transition between regimes: for $\mathrm{Re}<14$ laminar and waves regimes were observed, for $14 \leq \mathrm{Re} \leq 40$ the breaking-waves regime was detected, while the turbulent regime occurred for $\operatorname{Re}>40$.

Table 2 shows the frequency of occurrence of the different flow regimes in the present study for selected intervals of the Reynolds number. A laminar or a waves regime developed in $\sim 90 \%$ of the experiments having $\operatorname{Re}<39 ; 80 \%$ of the experiments with $39 \leq \mathrm{Re} \leq 85$ behaved in a breaking-waves regime, while for $\operatorname{Re}>85$, $\sim 95 \%$ of the experiments displayed a turbulent regime. Thus, for dense currents flowing over and/or through a rough bottom, larger Re than those observed for dense currents propagating on a smooth bottom have to be reached in order to observe the passage through the different flow regimes. To characterize the different regimes including the influence of the Froude number, we determined the transition between regimes using the buoyancy Reynolds number $\mathrm{Fr}^{2} \mathrm{Re}$. This nondimensional number is an important parameter widely used to describe stratified, turbulent mixing (Riley and de Bruyn Kops 2003; Hebert and de Bruyn Kops 2006).
The buoyancy Reynolds number must be above a critical value, typically taken to be $25-30$, for stratified turbulence mixing to be active. The frequency of occurrence of the flow regimes in terms of $\mathrm{Fr}^{2} \mathrm{Re}$ is as follows: laminar and waves regimes were generally observed for $\mathrm{Fr}^{2} \mathrm{Re}<50$ (in $\sim 80 \%$ of the experiments), the breaking-waves regime developed for $50 \leq \mathrm{Fr}^{2} \mathrm{Re} \leq$ 180 (in $75 \%$ of the experiments), and fully developed turbulent patterns were observed for $\mathrm{Fr}^{2} \mathrm{Re}>180$ (in $\sim 90 \%$ of the experiments).

\section{Entrainment dependence on slope angle and roughness height}

The inclination of the sloping bottom is an important parameter that strongly affects the current velocity and hence the flow dynamics. Different flow regimes were observed to develop when varying $s$, with relevant consequences on the entrainment processes. For small values of $s(0.4 \leq s \leq 0.85)$ the laminar and the nonbreaking-waves regimes were observed in $\sim 90 \%$ of the experiments for all values of $a h$ tested, and, as expected, the entrainment in these regimes was generally lower than for the other regimes (Fig. 3). In these regimes the

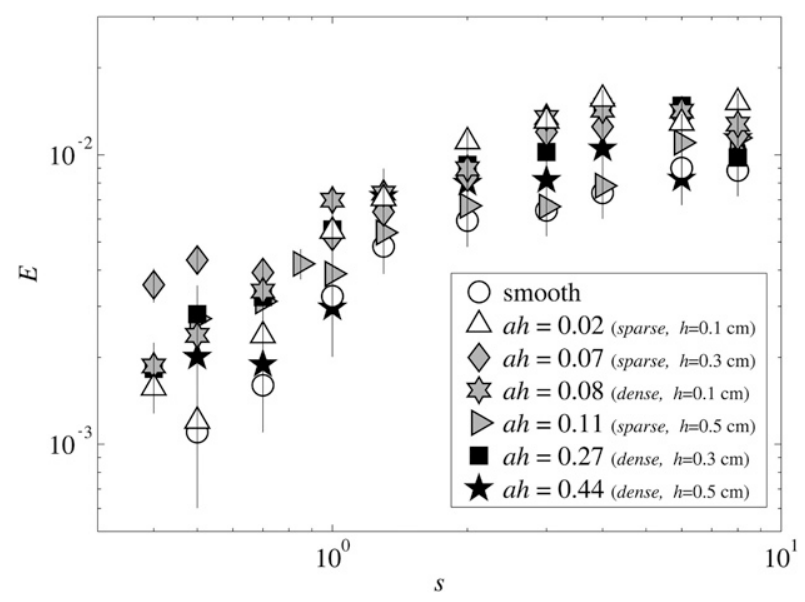

FIG. 3. Entrainment parameter dependence on the slope angle $s=\tan \theta$. Smooth bottom experiments are indicated by the open circles. White symbols are used for the low-value ah cases, gray symbols represent the intermediate-value ah cases, and black symbols mark the high-value $a h$ cases. Error bars are calculated as described in section 3 . 


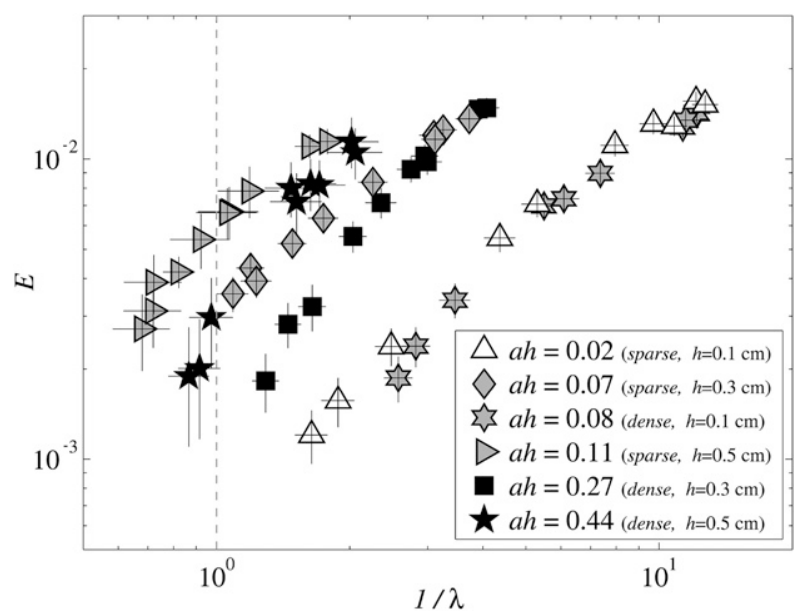

FIG. 4. Entrainment parameter dependence on the ratio of dense current height to roughness elements height $1 / \lambda$. Symbols as in Fig. 3. Error bars are calculated as described in section 3. Vertical dashed line indicates when $h=H_{m}$.

interface between the dense and the light fluids is sharp and only slightly perturbed by the waves; hence, a low amount of ambient fluid is entrained in the dense current. For intermediate values of $s(1 \leq s \leq 3)$, the breaking-waves regime was usually observed (in $\sim 65 \%$ of the experiments) independently of the roughness parameter $a h$. This regime is associated with a large amount of entrainment (Fig. 3) caused by the wave crests breaking and propagating downstream with the current (Cenedese and Adduce 2008). Finally, for high inclinations of the slope ( $s \geq 4)$, a fully developed turbulent regime was observed in $\sim 90 \%$ of the experiments, and the highest values of $E$ are distinctly detectable and are approximately constant for increasing values of $s$, confirming that a fully developed turbulent regime has been reached (Fig. 3).

Although the behavior described above was observed for all of the different roughness configurations, some differences in entrainment were also observed for varying $a h$ and $\lambda$. In Fig. 3 and subsequent figures, white symbols mark the low-value $a h$ cases, gray markers are used for the intermediatevalue $a h$ cases, and black symbols represent the highvalue $a h$ cases. The experiments over a smooth bottom (Fig. 3, open circles) are generally characterized by the lowest values of $E$ for all slopes. For $s<1$ the largest values of $E$ are usually observed in the intermediate roughness cases (gray symbols in Fig. 3), while the lowest values of $E$ are associated with the lowest $a h=$ 0.02 , the sparse configuration case in which the elements are, in general, much lower than the dense current height (Fig. 4). For $s \geq 1$, the largest entrainment is, in general, detected in the $h=0.1 \mathrm{~cm}$ cases (white triangles and gray stars in Fig. 3).

The ratio between the height of the cylinders and the height of the current $\lambda=h / H_{m}$ represents another important parameter. According to Eq. (5), an increase of the entrainment $E$ leads to an increase in $H_{m}$ and thereby a decrease in $\lambda$ for a fixed value of $h$ (Fig. 4). For fixed values of $h$, the sparse roughness configurations $(\Delta S=1.6 \mathrm{~cm})$ are in general characterized by lower heights of the gravity current than the corresponding dense configuration case $(\Delta S=0.8 \mathrm{~cm})$. This behavior can be explained both by the lower value of $V_{c}$ for the sparse configuration that decreases $H_{m}$ [Eq. (5)] and by the fact that in the sparse configuration the current flows downslope, propagating between the roughness elements, while for the dense configuration the current rides on top of the cylinders and mixes with the lighter fluid between the cylinders (Cenedese et al. 2017), consequently increasing the total current height. In some of the cases with $h=0.5 \mathrm{~cm}$ the height of the current is even lower than the roughness elements, that is, $1 / \lambda<1$ (gray triangles and black stars in Fig. 4). However, for approximately $1 / \lambda \geqslant 3$, the difference in $H_{m}$ between the experiments having the same $h$ in dense and sparse configurations is negligible. For these experiments the current is significantly higher than the roughness elements, and the difference in $V_{c}$ between the dense and sparse configuration is negligible compared to the total volume of the current $V$, producing a similar value of $H_{m}$, as indicated by Eq. (5).

The maximum values of $E$ for each configuration are observed to increase with decreasing $\lambda$ (Fig. 4). In particular, the highest values of the entrainment are $E \sim$ 0.011 for $h=0.3 \mathrm{~cm}$ and $E \sim 0.016$ for $h=0.1 \mathrm{~cm}$. With the exception of a few experiments having $h=0.1 \mathrm{~cm}$, in general the height of the current is comparable to that of the roughness elements, that is, $\lambda \sim 1$. Hence, we expect the vortices generated in the wake of the cylinders in the sparse configuration to enhance the entrainment of ambient water into the current. It is important to note that the values of $E$ in the present experiments are smaller than those found in similar experiments investigating entrainment in a dense current flowing down a smooth bottom (e.g., Ellison and Turner 1959; Parker et al. 1987; Cenedese and Adduce 2008) due to the reduced values of the Reynolds number.

\section{Entrainment dependence on Froude and Reynolds numbers}

The dependence of the entrainment parameter on $\mathrm{Fr}$ is shown in Fig. 5a, where, in general, larger values of $E$ are observed for larger $\mathrm{Fr}$, for a constant value of $a h$. 
(a)

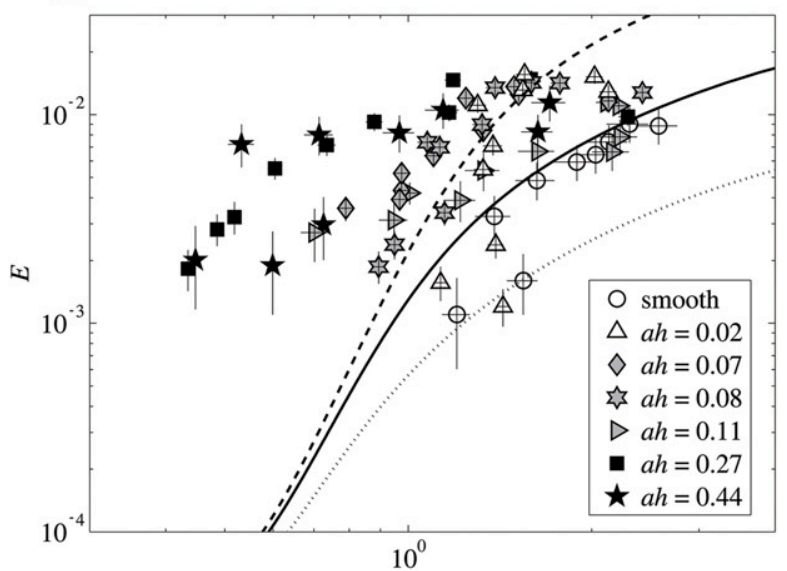

(b)

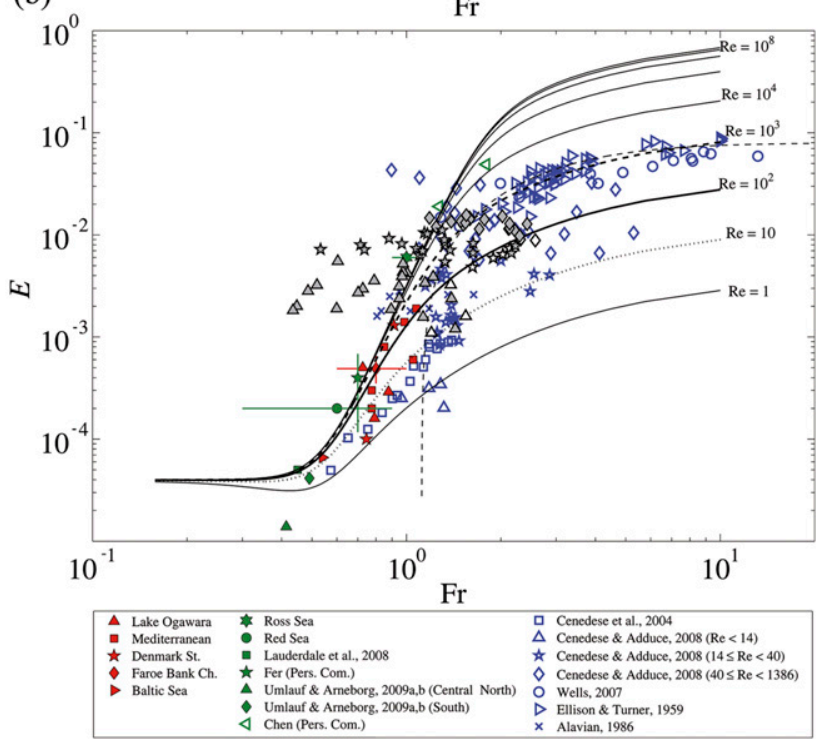

FIG. 5. Entrainment parameter dependence on the Froude number. (a) Symbols as in Fig. 3. Error bars are calculated as described in section 3. Dotted, solid, and dashed lines represent the entrainment parameterization for dense currents over a smooth bottom of Cenedese and Adduce (2010) for $\mathrm{Re}=10,100$, and 1000, respectively. (b) Figure adapted from Fig. 2 of Cenedese and Adduce (2010). The thin dashed line represents the Turner (1986) entrainment parameterization. Blue open and red solid symbols are the laboratory and field data, respectively, used to obtain the coefficients of Cenedese and Adduce (2010) entrainment parameterization represented by the thin and thick lines, where the thick lines are the same lines as in Fig. 5a. Green symbols are additional data used by Cenedese and Adduce (2010) to test their parameterization. The solid gray and open black symbols are the same data as in Fig. 5a for the rough and smooth bottom, respectively. The different symbols represent different ranges of $\mathrm{Re} ; \mathrm{Re}<39$ (triangles), $39 \leq \operatorname{Re} \leq 85$ (stars), and $\operatorname{Re}>85$ (squares).

However, the data in Fig. 5a show a considerable scatter that can be partially explained when looking at the different values of $a h$ (indicated by the different symbols in Fig. 5a). Looking at the white, gray, and black symbols in Fig. 5a, representing the small, intermediate, and large values of $a h$, respectively, one can notice that while the Fr decreases with increasing $a h$, the range of values of $E$ is approximately the same, that is, black symbols are shifted toward the left compared to the white symbols in the Fr axis but are approximately at the same location on the $E$ axis. As discussed in section 9, this can be explained by the different mechanisms at play associated with different values of $a h$.

The large scatter between Fr and $E$ visible in Fig. 5a is also due to the different Reynolds numbers, as discussed previously in Cenedese and Adduce (2008). For a fixed Fr, larger values of $E$ are observed as Re increases. For example, for $\mathrm{Fr} \sim 1.2, \operatorname{Re} \sim 13$ in the smooth case (white circle at the bottom of the figure), $\operatorname{Re} \sim 33$ in the $a h=$ 0.11 case (gray right side triangle), and $\mathrm{Re} \sim 110$ in the $a h=0.27$ case (black square at the top of the figure).

An almost linear dependence of $E$ on Re has been observed (Fig. 6), in agreement with Cenedese and Adduce (2008). The largest values of Re and $E$ are observed for $a h=0.02$ and 0.08 , which are the roughness values for $h=0.1 \mathrm{~cm}$. As expected, the largest $E$ are associated with the turbulent regime observed for $\mathrm{Re}>$ 85 and for the wave-breaking regime observed for $39 \leq$ $\mathrm{Re} \leq 85$ (black dashed lines in Fig. 6).

While Fig. 5a clearly shows that the Froude number generally decreases as $a h$ increases, due in most cases to a decrease of $U_{m}$, the Reynolds number is not clearly sensitive to the value of $a h$, as all values of ah span a similar range in Reynolds number (Fig. 6). The independence of the Reynolds number on the roughness parameter $a h$ can be explained by the fact that for large $a h$ the bulk velocity is observed to be lower than that for high $a h$, while, in general, the value of $H_{m}$ is larger and hence resulting in a value of the Reynolds number that is not sensitive to the roughness parameter.

Furthermore, the value of the entrainment parameter is more sensitive to the value of $\mathrm{Re}$ than Fr, that is, Fig. $5 \mathrm{~b}$ shows that similar values of $E$ are associated with similar values of $\mathrm{Re}$, while the Froude number varies from a factor of 2 for the highest Re (gray squares in Fig. 5b) to more than half an order of magnitude for the lowest values of Re (gray triangles in Fig. 5b). This weaker dependence of the entrainment parameter on $\mathrm{Fr}$ is not as prominent for a dense current flowing over a smooth bottom, as illustrated 


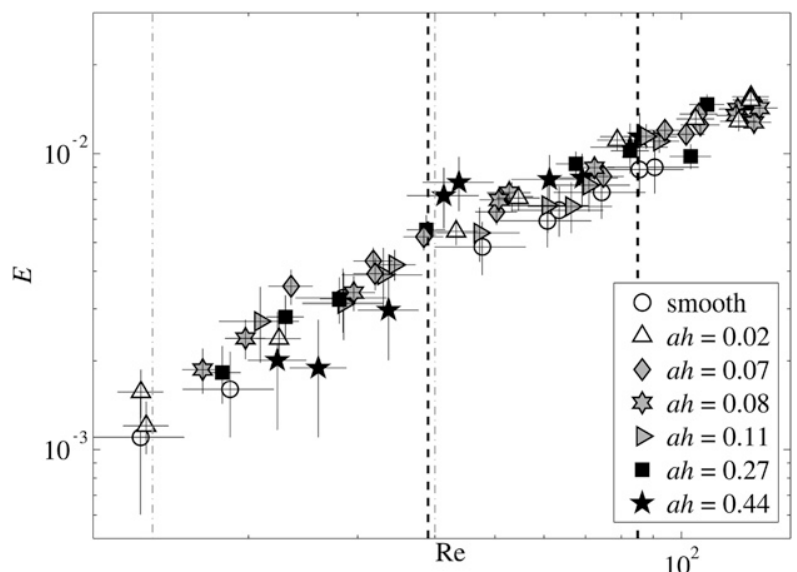

FIG. 6. Entrainment parameter dependence on the Reynolds number. Symbols as in Fig. 3. Error bars are calculated as in section 3. Black dashed lines are for $\mathrm{Re}=39$ and 85 and divide the different flow regimes for rough bottom experiments, while gray dashed-dotted lines are for $\operatorname{Re}=14$ and 40, which are the limits between different regimes for the smooth experiments of Cenedese and Adduce (2008).

by our data and the data of Cenedese and Adduce (2008) in Fig. 5b. The value of $E$ can be the same for different values of Fr also for a dense current flowing over a smooth bottom; however, the ranges of Fr where this is observed are much smaller than in the case of a dense current over a rough bottom, especially for the lowest values of Fr. For large $\mathrm{Fr}$, that is, $\mathrm{Fr}>2$, the parameterizations of Turner (1986) and Cenedese and Adduce (2010) suggest that $E$ is weakly dependent on Fr, as indicated by the flattening of the curves in Fig. 5.

A possible explanation for the (low Fr) weaker dependence of the entrainment parameter on $\mathrm{Fr}$ is that, in the presence of a rough bottom, the entrainment process is influenced not only by shear instability due to density differences, but some entrainment occurs also via the vortices created in the wake of the cylinders and convective mixing [as observed by Cenedese et al. (2017) and discussed in section 5]. The above mechanisms for entrainment are expected to be more effective near the head of the current (Cenedese et al. 2017), while our measurements are representative of the entrainment occurring once a quasi-steady state regime is reached in the body of the current. Hence, we expect our entrainment data to be influenced both by the above mechanisms and also by shear instability, retaining a small, and possibly not as clear, dependence of the entrainment parameter on the Froude number.

To consider the influence of both Fr and Re, the entrainment parameter versus the buoyancy Reynolds number $\mathrm{Fr}^{2} \mathrm{Re}$ is plotted in Fig. 7, where the increase of $E$ at the increase of $\mathrm{Fr}^{2} \mathrm{Re}$ for each $a h$ is clearly visible.

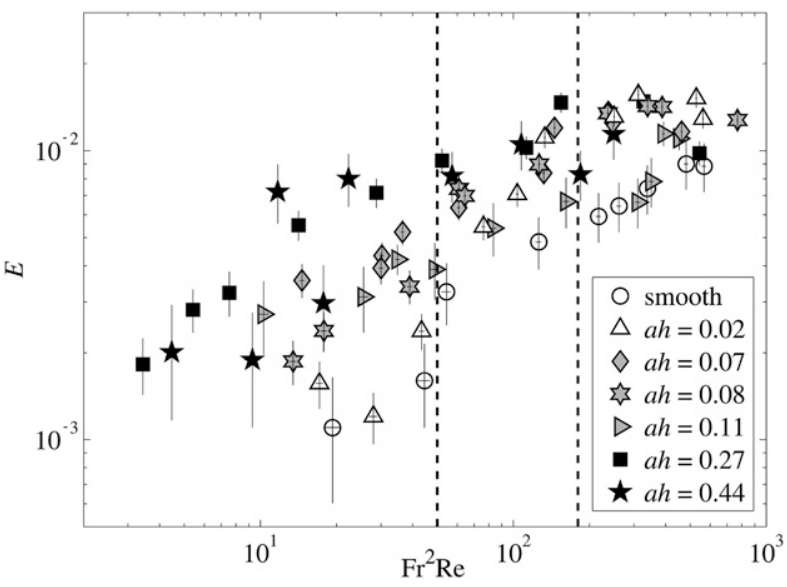

FIG. 7. Entrainment parameter dependence on $\mathrm{Fr}^{2} \mathrm{Re}$. Symbols as in Fig. 3. Error bars are calculated as in section 3. Black dashed lines show $\mathrm{Fr}^{2} \mathrm{Re}=50$ and 180, dividing the different flow regimes for the rough bottom experiments.

For fixed values of $\mathrm{Fr}^{2} \mathrm{Re}$, the smooth and the low ah cases (white markers) are characterized by lower $E$ than the intermediate $a h$ cases (gray markers) and the high $a h$ cases (black markers). As previously observed in Fig. 6, Fig. 7 shows the largest values of $E$ occurring in the $a h=$ 0.02 and 0.08 cases, which, in general, are characterized by the largest value of $\mathrm{Fr}^{2} \mathrm{Re}$.

For low and intermediate values of $\mathrm{Fr}^{2} \mathrm{Re}$, the increase of $E$ at the increase of $a h$ for a fixed value of $\mathrm{Fr}^{2} \mathrm{Re}$ is clearly visible, while for large values of $\mathrm{Fr}^{2} \mathrm{Re}$, a fully developed turbulent regime occurs and high values of $E$ are always detected independently of $a h$, albeit $E$ is increased compared to the smooth bottom cases. Finally, given the observed independence of the Reynolds number on the value of $a h$ (Fig. 6), the decrease of $\mathrm{Fr}^{2} \mathrm{Re}$ with the increase in $a h$ is due to the decrease in Fr with increasing $a h$ observed in Fig. 5a.

\section{Discussion}

The results illustrated in the previous sections clearly show that the presence of roughness elements strongly influences the dynamics of a dense current flowing down a sloping bottom, both in terms of the dense current velocity and the entrainment of ambient waters. The presence of a rough bottom enhances the drag exerted on the dense current (e.g., Tanino et al. 2005; Tanino and Nepf 2008; Zhang and Nepf 2008) with the consequent decrease in the dense current velocity $U_{m}$. Hence, for fixed values of $Q_{0}, g_{0}^{\prime}$, and $s$ an increase in $a h$ caused a reduction of the Froude number, while the Reynolds number was found to be generally insensitive to the value of $a h$ due to the increase of $H_{m}$ 
associated with the decrease in $U_{m}$ observed for large values of $a h$.

Previous studies (e.g., Ellison and Turner 1959; Cenedese and Adduce 2008) suggest that the magnitude of the entrainment is uniquely related to the $\mathrm{Fr}$ and $\mathrm{Re}$ of the flow, a concept that allows the development of parameterizations for entrainment based on the relevant nondimensional parameters (Fig. 5, dotted, solid, and dashed lines). However, our results suggest that this may not be the case in the presence of a rough bottom (e.g., Fig. 5), indicating that additional nondimensional parameters may need to be considered to correctly parameterize entrainment when a rough bottom is present. Additional nondimensional parameters are necessary to take into consideration the new dynamics affecting entrainment that arise in the presence of a rough bottom. In particular, the presence of the roughness elements has been shown to inhibit the formation or the growth of the turbulent structures generated by shear instability at the interface between the dense and ambient fluids (Negretti et al. 2008), and entrainment can be enhanced by the vortices generated in the wake of the cylinders in a sparse configuration or by the convective instability occurring in a dense configuration (Cenedese et al. 2017).

The reduction of the growth rate of the turbulent structures generated by shear instability at the interface between the dense and ambient fluids (Negretti et al. 2008) may explain why the transition between the different flows regimes for a dense current over a rough bottom occurred for larger Re than those observed in the smooth bottom experiments (section 6). We also expect that this reduction is going to be larger for increasing values of $h$. Hence, in general, the largest values of $E$ are observed for the experiments having $h=0.1 \mathrm{~cm}$, or lower $a h$ (Figs. 3, 7), because the small height of the cylinders does not inhibit the formation of turbulent disturbances at the interface between the two fluids, and the breaking-waves and the turbulent regimes can develop; nevertheless, the presence of the roughness slightly increases the values of the entrainment when compared to the smooth cases. When in the laminar and nonbreaking-waves regimes, the opposite happens: the lowest entrainment is observed for the lowest value of $a h$ because shear instability is not occurring, and the roughness elements, being too small, do not substantially enhance entrainment compared with the smooth bottom cases.

The decrease in the dense current velocity due to bottom drag explains the decrease in $\mathrm{Fr}$ and $\mathrm{Fr}^{2} \mathrm{Re}$ with increasing $a h$, that is, the horizontal shift of the symbols having the same color in Figs. 5a and 7, while the different mechanisms enhancing entrainment observed by Cenedese et al. (2017) can clarify why the range of $E$ is approximately the same for all $a h$. In the presence of a dense rough bottom (i.e., larger values of $a h$ ), the current is strongly decelerated with a consequent reduction of the Froude number. However, the convective instability arising within the roughness elements (Cenedese et al. 2017) produces a relatively large $E$ for such low $\mathrm{Fr}$ or $\mathrm{Fr}^{2} \mathrm{Re}$ when compared with previous experiments. In Fig. 5a the parameterization proposed by Cenedese and Adduce (2010) is indicated by the dotted, solid, and dashed lines for $\operatorname{Re}=10,100$, and 1000 , respectively. These curves were obtained by fitting an extensive dataset of laboratory experiments conducted in this range of $\mathrm{Re}$ for dense currents over a smooth bottom (Fig. 5b). Figure 5b shows that for low $\mathrm{Fr}$ the value of $E$ in the presence of a rough bottom (gray solid symbols) is significantly larger than for the smooth bottom experiments (blue open symbols) parameterized by the dotted, solid, and dashed lines. The gray symbols in Figs. 5a and 7 have, in general, lower Fr and $\mathrm{Fr}^{2} \mathrm{Re}$ than the smooth cases and white symbols due to the enhanced drag generated by the roughness elements. In these experiments entrainment is likely enhanced by the vortices generated in the wake of the cylinders, and the range of values for $E$ is comparable to that in the experiments having larger Fr or $\mathrm{Fr}^{2} \mathrm{Re}$ (Figs. 5a, 7).

In summary, the presence of a rough bottom affects the dynamics of the dense current by both decreasing the velocity of the flow, and consequently the Froude number (Figs. 5, 7), and by increasing the entrainment, for fixed values of $\mathrm{Fr}^{2} \mathrm{Re}$, with increasing $a h$ due to two different mechanisms: the vortices generated in the wake of the cylinders enhancing the entrainment of ambient waters through the current interface (subset of gray symbols) and the convective instability mixing the ambient waters between the cylinders with the dense current moving on top of it (black symbols).

In the present study, the effect of rotation on the entrainment was not investigated. We choose to reproduce the experiment of Cenedese and Adduce (2008) and focus exclusively on the effects of a rough bottom on the dynamics regulating the entrainment, while keeping the Coriolis parameter constant. Previous laboratory studies (e.g., Cenedese et al. 2004) showed that rotational effects can cause the generation of cyclonic eddies above the dense current, but the entrainment in this eddy regime was small when compared to the entrainment in dense currents in the wave or turbulent regimes (Cenedese and Adduce 2008). Rotational effects strongly modify the trajectory of the dense current and the current velocity, ultimately influencing the values of Fr and Re (section 1). However, as discussed in detail in Cenedese and Adduce (2010), we believe that when a dense current descends a slope, that is, not in a submarine channel, "local" mixing is not affected by rotation directly, given that the turnover time scale 
of the turbulent eddies is much faster than the inertial period. The rotating experiments of Wells (2007) show entrainment values almost indistinguishable from those obtained in the nonrotating experiments of Ellison and Turner (1959), providing supporting evidence for this assumption.

\section{Summary and conclusions}

The entrainment in a dense current flowing down through and/or over a rough bottom in a rotating system was investigated using laboratory experiments. The influence on the entrainment of the slope angle and the spacing and the height of the bottom roughness elements were investigated. The entrainment parameter dependence on the relevant dimensionless numbers, that is, the slope $s$, the ratio $\lambda$, the Froude and Reynolds numbers, and the buoyancy Reynolds number $\mathrm{Fr}^{2} \mathrm{Re}$ was discussed. As in Cenedese et al. (2017), in the sparse configuration the dense current propagated between the cylinders. For a dense configuration, we could not observe the flow between the cylinders, and we assume that the dense current moved on top of the cylinders above lighter water and was subjected to convective instability with consequent enhancement of the current dilution and entrainment of ambient waters, as observed in Cenedese et al. (2017).

Previous studies suggest that the presence of a rough bottom affects the dynamics of the dense current and the entrainment through the development of two contrasting mechanisms: the rough elements enhance the drag exerted on the current and cause a decrease in current velocity, with an associated reduction of the Froude number, and inhibit the formation or growth of turbulent disturbances at the interface, reducing the entrainment; at the same time, the additional turbulence related to the presence of the roughness elements acts to enhance the entrainment. The latter is in the form of vortices in the wake of the cylinders, enhancing the entrainment of ambient waters through the current interface, for a sparse configuration, and convective instability mixing the dense current above the cylinders with the lighter water between them, for a dense configuration.

An increase of $E$ with the increase of $s$ was observed because of the transition through the flow regimes previously observed by Cenedese and Adduce (2008). For a fixed value of $s$, different values of the entrainment parameter were observed by varying ah (Fig. 3) because different Fr and Re numbers characterized the flow. The ratio between the current height and the height of the roughness elements was $O(1)$ for most experiments, suggesting that in the sparse configuration the vortices generated in the wake of the cylinders can enhance entrainment by engulfing ambient waters into the dense current.

Following the approach of Cenedese and Adduce (2008), selected values of $\mathrm{Re}$ and $\mathrm{Fr}^{2} \mathrm{Re}$ were chosen as thresholds to mark the occurrence of the different flow regimes, and new thresholds were identified for dense currents flowing down a rough bottom. It was found that the passage through the different flow regimes occurs for larger $\mathrm{Re}$ when compared to dense currents flowing down a smooth bottom, possibly because the formation of turbulent disturbances at the interface between the two layers is inhibited by the bottom roughness. The $\mathrm{Fr}$ number was observed to decrease with increasing ah because of the enhanced drag and consequent decrease of the velocity of the dense current. Nevertheless, relatively high values of $E$ were detected also for $\mathrm{Fr}<1$ cases because of the additional turbulence developed in the bottom roughness region, mainly caused by convective instability. This is qualitatively in agreement with the results of Fernandez and Imberger (2006), which showed that for large Richardson numbers $(\mathrm{Ri}>10)$ the entrainment rate was larger than that predicted by parameterization based only on Ri. Finally, the amount of ambient fluid entrained in the dense current was observed to increase with the increase of the buoyancy Reynolds number $\mathrm{Fr}^{2} \mathrm{Re}$. For a fixed $\mathrm{Fr}^{2} \mathrm{Re}, E$ was observed to increase with increasing $a h$. However, for larger values of $\mathrm{Fr}^{2} \mathrm{Re}$, in the turbulent regime, the large entrainment due to shear instability was only mildly enhanced by the rough bottom, and experiments with different $a h$ present similar values of $E$.

Acknowledgments. The authors thank Anders Jensen for his invaluable technical expertise in constructing the experimental apparatus and Jason Hyatt for helpful comments on the manuscript. Support to C. C. was given by the National Science Foundation Project OCE1333174. Support to L. O. during her internship at WHOI was provided by the Lions Club "Napoli Megaride" and the Zoological Station Anton Dohrn through the Paolo Brancaccio fellowship (2012).

\section{REFERENCES}

Cenedese, C., and C. Adduce, 2008: Mixing in a density-driven current flowing down a slope in a rotating fluid. J. Fluid Mech., 604, 369-388, doi:10.1017/S0022112008001237.

- and - 2010: A new parameterization for entrainment in overflows. J. Phys. Oceanogr., 40, 1835-1850, doi:10.1175/ 2010JPO4374.1.

_ J. A. Whitehead, T. Ascarelli, and M. Ohiwa, 2004: A dense current flowing down a sloping bottom in a rotating fluid. J. Phys. Oceanogr., 34, 188-203, doi:10.1175/1520-0485(2004)034<0188: ADCFDA $>2.0 . \mathrm{CO} ; 2$. 
_, R. Nokes, and J. Hyatt, 2017: Lock-exchange gravity currents over rough bottoms. Environ. Fluid Mech., doi:10.1007/ s10652-016-9501-0, in press.

Chang, Y., X. Xu, T. Özgökmen, E. Chassignet, H. Peters, and P. Fischer, 2005: Comparison of gravity current mixing parameterizations and calibration using a high-resolution 3D nonhydrostatic spectral element model. Ocean Modell., 10, 342-368, doi:10.1016/j.ocemod.2004.11.002.

Cossu, R., and M. G. Wells, 2010: Coriolis forces influence the secondary circulation of gravity currents flowing in large-scale sinuous submarine channel systems. Geophys. Res. Lett., 37, L17603, doi:10.1029/2010GL044296.

— _ — , and A. Wåhlin, 2010: Influence of the Coriolis force on the velocity structure of gravity currents in straight submarine channel systems. J. Geophys. Res. Oceans, 115, C11016, doi:10.1029/2010JC006208.

Dallimore, C. J., J. Imberger, and T. Ishikawa, 2001: Entrainment and turbulence in saline underflow in Lake Ogawara. J. Hydraul. Eng., 127, 937-948, doi:10.1061/ (ASCE)0733-9429(2001)127:11(937).

Ellison, T. H., and J. S. Turner, 1959: Turbulent entrainment in stratified flows. J. Fluid Mech., 6, 423-448, doi:10.1017/ S0022112059000738.

Ezer, T., 2005: Entrainment, diapycnal mixing and transport in three-dimensional bottom gravity current simulations using the Mellor-Yamada turbulence scheme. Ocean Modell., 9, 151-168, doi:10.1016/j.ocemod.2004.06.001.

Fernandez, R. L., and J. Imberger, 2006: Bed roughness induced entrainment in a high Richardson number underflow. J. Hydraul. Res., 44, 725-738, doi:10.1080/00221686.2006.9521724.

Girton, J. B., and T. B. Sanford, 2003: Descent and modification of the overflow plume in the Denmark Strait. J. Phys. Oceanogr., 33, 1351-1364, doi:10.1175/1520-0485(2003)033<1351: DAMOTO $>2.0 . \mathrm{CO} ; 2$.

Hacker, J., P. Linden, and S. Dalziel, 1996: Mixing in lock-release gravity currents. Dyn. Atmos. Oceans, 24, 183-195, doi:10.1016/0377-0265(95)00443-2.

Hanquiez, V., T. Mulder, P. Lecroart, E. Gonthier, E. Marchès, and M. Voisset, 2007: High resolution seafloor images in the Gulf of Cadiz, Iberian margin. Mar. Geol., 246, 42-59, doi:10.1016/ j.margeo.2007.08.002.

Hebbert, B., J. Patterson, I. Loh, and J. Imberger, 1979: Collie river underflow into the Wellington Reservoir. J. Hydraul. Div., 105, 533-545.

Hebert, D. A., and S. M. de Bruyn Kops, 2006: Predicting turbulence in flows with strong stable stratification. Phys. Fluids, $\mathbf{1 8}$, 066602, doi:10.1063/1.2204987.

Hernández-Molina, F. J., and Coauthors, 2006: The contourite depositional system of the Gulf of Cadiz: A sedimentary model related to the bottom current activity of the Mediterranean outflow water and its interaction with the continental margin. DeepSea Res. II, 53, 1420-1463, doi:10.1016/j.dsr2.2006.04.016.

Keeton, J., R. Searle, C. Peirce, B. Parsons, R. White, M. Sinha, B. Murton, and L. Parson, 1997: Bathymetry of the Reykjanes Ridge. Mar. Geophys. Res., 19, 55-64, doi:10.1023/A:1004266721393.

Legg, S., R. Hallberg, and J. Girton, 2006: Comparison of entrainment in overflows simulated by z-coordinate, isopycnal and non-hydrostatic models. Ocean Modell., 11, 69-97, doi:10.1016/j.ocemod.2004.11.006.

Luhar, M., J. Rominger, and H. Nepf, 2008: Interaction between flow, transport and vegetation spatial structure. Environ. Fluid Mech., 8, 423-439, doi:10.1007/s10652-008-9080-9.
Mauritzen, C., J. Price, T. Sanford, and D. Torres, 2005: Circulation and mixing in the Faroese Channels. Deep-Sea Res. I, 52, 883913, doi:10.1016/j.dsr.2004.11.018.

Negretti, M., D. Zhu, and G. Jirka, 2008: The effect of bottom roughness in two-layer flows down a slope. Dyn. Atmos. Oceans, 45, 46-68, doi:10.1016/j.dynatmoce.2008.02.001.

Nepf, H., 2012: Flow and transport in regions with aquatic vegetation. Annu. Rev. Fluid Mech., 44, 123-142, doi:10.1146/ annurev-fluid-120710-101048.

Nogueira, H., C. Adduce, E. Alves, and M. Franca, 2013: Analysis of lock-exchange gravity currents over smooth and rough beds. J. Hydraul. Res., 51, 417-431, doi:10.1080/ 00221686.2013 .798363$.

$\_,-,-,$and -2014 : Dynamics of the head of gravity currents. Environ. Fluid Mech., 14, 519-540, doi:10.1007/ s10652-013-9315-2.

Noh, Y., and H. Fernando, 1991: Gravity current propagation along an incline in the presence of boundary mixing. J. Geophys. Res. Oceans, 96, 12 586-12 592, doi:10.1029/90JC02488.

$\longrightarrow$, and - 1992: The motion of a buoyant cloud along an incline in the presence of boundary mixing. J. Fluid Mech., 235 , 557-577, doi:10.1017/S0022112092001228.

Ottolenghi, L., C. Adduce, R. Inghilesi, V. Armenio, and F. Roman, 2016a: Entrainment and mixing in unsteady gravity currents. J. Hydraul. Res., 54, 541-557, doi:10.1080/ 00221686.2016 .1174961$.

,,,--- F. Roman, and V. Armenio, 2016b: Mixing in lock-release gravity currents propagating up a slope. Phys. Fluids, 28, 056604, doi:10.1063/1.4948760.

Özgökmen, T. M., and P. F. Fischer, 2008: On the role of bottom roughness in overflows. Ocean Modell., 20, 336-361, doi:10.1016/j.ocemod.2007.10.004.

_ - J. Duan, and T. Iliescu, 2004: Entrainment in bottom gravity currents over complex topography from threedimensional nonhydrostatic simulations. Geophys. Res. Lett., 31, L13212, doi:10.1029/2004GL020186.

Özgökmen, T., T. Iliescu, and P. Fischer, 2009: Reynolds number dependence of mixing in a lock-exchange system from direct numerical and large eddy simulations. Ocean Modell., 30, 190206, doi:10.1016/j.ocemod.2009.06.013.

Parker, G., M. Garcia, Y. Fukushima, and W. Yu, 1987: Experiments on turbidity currents over an erodible bed. J. Hydraul. Res., 25, 123-147, doi:10.1080/00221688709499292.

Peters, H., W. E. Johns, A. S. Bower, and D. M. Fratantoni, 2005: Mixing and entrainment in the red sea outflow plume. Part I: Plume structure. J. Phys. Oceanogr., 35, 569-583, doi:10.1175/ JPO2679.1.

Price, J. F., and M. O. Barringer, 1994: Outflows and deep water production by marginal seas. Prog. Oceanogr., 33, 161-200, doi:10.1016/0079-6611(94)90027-2.

_ , and Coauthors, 1993: Mediterranean outflow mixing and dynamics. Science, 259, 1277-1282, doi:10.1126/science.259.5099.1277.

Riley, J. J., and S. M. de Bruyn Kops, 2003: Dynamics of turbulence strongly influenced by buoyancy. Phys. Fluids, 15, 2047-2059, doi:10.1063/1.1578077.

Sherwin, T. J., and W. R. Turrell, 2005: Mixing and advection of a cold water cascade over the Wyville Thomson Ridge. DeepSea Res. I, 52, 1392-1413, doi:10.1016/j.dsr.2005.03.002.

Simpson, J. E., 1997: Gravity Currents in the Environment and the Laboratory. 2nd ed. Cambridge University Press, $244 \mathrm{pp}$.

Tanino, Y., and H. M. Nepf, 2008: Laboratory investigation of mean drag in a random array of rigid, emergent cylinders. J. Hydraul. Eng., 134,34-41, doi:10.1061/(ASCE)0733-9429(2008)134:1(34). 
— - — and P. S. Kulis, 2005: Gravity currents in aquatic canopies. Water Resour. Res., 41, W12402, doi:10.1029/ 2005 WR004216.

Turner, J. S., 1986: Turbulent entrainment: The development of the entrainment assumption and its application to geophysical flows. J. Fluid Mech., 173, 431-471, doi:10.1017/ S0022112086001222.

Umlauf, L., and L. Arneborg, 2009a: Dynamics of rotating shallow gravity currents passing through a channel. Part I: Observation of transverse structure. J. Phys. Oceanogr., 39, 2385-2401, doi:10.1175/2009JPO4159.1.

— rents passing through a channel. Part II: Analysis. J. Phys. Oceanogr., 39, 2402-2416, doi:10.1175/2009JPO4164.1.

,,-- R. Hofmeister, and H. Burchard, 2010: Entrainment in shallow rotating gravity currents: A modeling study. J. Phys. Oceanogr., 40, 1819-1834, doi:10.1175/2010JPO4367.1.
Wåhlin, A., E. Darelius, C. Cenedese, and G. Lane-Serff, 2008: Laboratory observations of enhanced entrainment in dense overflows in the presence of submarine canyons and ridges. Deep-Sea Res. I, 55, 737-750, doi:10.1016/j.dsr.2008.02.007.

Wells, M., 2007: Influence of Coriolis forces on turbidity currents and sediment deposition. Particle-Laden Flow, Springer, 331-343.

— C. Cenedese, and C. Caulfield, 2010: The relationship between flux coefficient and entrainment ratio in density currents. J. Phys. Oceanogr., 40, 2713-2727, doi:10.1175/2010JPO4225.1.

Xu, X., Y. Chang, H. Peters, T. Özgökmen, and E. Chassignet, 2006: Parameterization of gravity current entrainment for ocean circulation models using a high-order 3D nonhydrostatic spectral element model. Ocean Modell., 14, 19-44, doi:10.1016/j.ocemod.2006.02.006.

Zhang, X., and H. M. Nepf, 2008: Density-driven exchange flow between open water and an aquatic canopy. Water Resour. Res., 44, W08417, doi:10.1029/2007WR006676. 